\title{
SCLIP Is Crucial for the Formation and Development of the Purkinje Cell Dendritic Arbor
}

\author{
Fabienne E. Poulain, ${ }^{1,2,4}$ Stéphanie Chauvin, ${ }^{1,2,4}$ Rosine Wehrlé, ${ }^{3,4}$ Mathieu Desclaux, ${ }^{4,5}$ Jacques Mallet, ${ }^{4,5}$ \\ Guilan Vodjdani, ${ }^{4,5}$ Isabelle Dusart, ${ }^{3,4 *}$ and André Sobel ${ }^{1,2,4 *}$ \\ ${ }^{1}$ Institut National de la Santé et de la Recherche Médicale, Unité Mixte de Recherche (UMR) 839, ${ }^{2}$ Institut du Fer à Moulin, ${ }^{3}$ Centre National de la Recherche \\ Scientifique (CNRS), UMR7102-Neurobiologie des Processus Adaptatifs, and ${ }^{4}$ Université Pierre et Marie Curie, Université Paris 06, F-75005 Paris, France, \\ and ${ }^{5}$ CNRS, UMR7091-Génétique Moléculaire de la Neurotransmission et des Processus Neurodégénératifs, F-75013 Paris, France
}

Cerebellar Purkinje cells elaborate one of the most complex dendritic arbors among neurons to integrate the numerous signals they receive from the cerebellum circuitry. Their dendritic differentiation undergoes successive, tightly regulated phases of development involving both regressive and growth events. Although many players regulating the late phases of Purkinje cell dendritogenesis have been identified, intracellular factors controlling earlier phases of dendritic development remain mostly unknown. In this study, we explored the biological properties and functions of SCLIP, a protein of the stathmin family, in Purkinje cell dendritic differentiation and cerebellum development. Unlike the other stathmins, SCLIP is strongly expressed in Purkinje cells during cerebellar development and accumulates in their dendritic processes at a critical period of their formation and outgrowth. To reveal SCLIP functions, we developed a lentiviralmediated approach on cerebellar organotypic cultures to inhibit or increase its expression in Purkinje cells in their tissue environment. Depletion of SCLIP promoted retraction of the Purkinje cell primitive process and then prevented the formation of new dendrites at early stages of postnatal development. It also prevented their elongation and branching at later phases of differentiation. Conversely, SCLIP overexpression promoted dendritic branching and development. Together, our results demonstrate for the first time that SCLIP is crucial for both the formation and proper development of Purkinje cell dendritic arbors. SCLIP appears thus as a novel and specific factor that controls the early phases of Purkinje cell dendritic differentiation during cerebellum development.

Key words: dendritogenesis; differentiation; stathmin; cerebellar organotypic cultures; lentiviral vector; cerebellum development

\section{Introduction}

Dendritogenesis is an important process of neuronal morphogenesis essential for the establishment of neuronal circuitry. However, the mechanisms underlying dendrite formation and development remain, in contrast to axonogenesis, poorly understood.

One of the most highly sophisticated dendritic trees is that of cerebellar Purkinje cells (PCs), which allows them to integrate numerous signals from the cerebellar circuitry. PC dendritogenesis occurs through successive, tightly regulated stages of maturation involving both regressive and growth events (Ramón y Cajal, 1911; Armengol and Sotelo, 1991). During the first post-

\footnotetext{
Received Dec. 31, 2007; revised June 9, 2008; accepted June 9, 2008.

This work was supported by Institut National de la Santé et de la Recherche Médicale, Centre National de la Recherche Scientifique, Université Pierre et Marie Curie, Fondation pour la Recherche Médicale, Association Française contre les Myopathies, and Association pour la Recherche sur la Sclérose en Plaques. We thank Drs. R. Hawkes, M. Bornens, and P. Skene for the generous gifts of anti-zebrin II, CTR433, and anti-GAP-43 antibodies, respectively, and Dr. P. Charneau for providing the pFlap lentiviral vector. We are grateful to Drs. P. Gaspar and R. M. Mège for critical reading of this manuscript. Fluorescence and confocal microscopy were performed in the Institut du Fer à Moulin Imaging facility.

*I.D. and A.S. contributed equally to this work.

Correspondence should be addressed to Dr. André Sobel, Institut National de la Santé et de la Recherche Médicale/Université Pierre et Marie Curie, Unité Mixte de RechercheS839, Institut du Fer à Moulin, 17 rue du Fer à Moulin, F-75005 Paris, France. E-mail: sobel@fer-a-moulin.inserm.fr.

DOI:10.1523/JNEUROSCI.1942-08.2008

Copyright $\odot 2008$ Society for Neuroscience $\quad$ 0270-6474/08/287387-12\$15.00/0
}

natal week in rodents, bipolar fusiform cells retract their primitive processes and extend numerous short perisomatic protrusions conferring them a "stellate" morphology. PCs then undergo a rapid phase of dendritic elongation and branching to elaborate their typical dendritic tree, with a single trunk splitting into numerous branches (Altman, 1972). Various factors have been implicated in the regulation of these late phases of dendritic development (Kapfhammer, 2004). However, those controlling early stages of dendritogenesis remain poorly characterized (Boukhtouche et al., 2006).

Stathmin family phosphoproteins including stathmin, SCG10, SCLIP, and RB3, have been proposed to play specific and complementary roles in the formation and maturation of the nervous system (Ozon et al., 1997, 1998, 1999; Curmi et al., 1999). They have been originally identified for their capacity to integrate and relay intracellular signaling pathways (Sobel, 1991), at least in part through tubulin binding and the control of microtubule dynamics (Belmont and Mitchison, 1996; Curmi et al., 1997; Steinmetz et al., 2000; Charbaut et al., 2001; Ravelli et al., 2004). Whereas stathmin is ubiquitous and cytosolic, the other proteins are essentially expressed in the nervous system and localized at the Golgi complex and vesicular structures along neurite shafts and in growth cones (Di Paolo et al., 1997; Gavet et al., 2002; Charbaut et al., 2005). Their expression during development and their regional and cellular distribution in the brain are 
differently regulated (Ozon et al., 1999). We recently demonstrated that SCLIP and its closest family member SCG10 regulate axon morphogenesis differently, controlling axonal branching and growth cone expansion, respectively (Poulain and Sobel, 2007). Stathmin and SCG10 have been shown also to be implicated in axon specification (Watabe-Uchida et al., 2006) and dendritic maturation (Ohkawa et al., 2007). Stathmin family proteins thus appear as essential regulators of neuronal morphogenesis that might play important and complementary functions in the control of cerebellar development.

In contrast to stathmin or SCG10, SCLIP mRNA is highly expressed in PCs (Ozon et al., 1999). It thus appears as a good candidate for regulating PC dendritogenesis, possibly at early stages of development. In the present study, we explored the biological properties of SCLIP during cerebellar development and investigated its potential role in the formation and development of PC dendritic arbors. We show that SCLIP protein is highly expressed in PCs during early development and specifically accumulates in their elongating dendrites at a critical period of outgrowth. We further demonstrate in cerebellar organotypic cultures that SCLIP plays a key role in the early steps of PC dendritic development, controlling both primitive process regression and new dendrite formation. Finally, SCLIP is also important for the regulation of dendritic elongation and branching at later phases of differentiation. Together, our study identifies for the first time SCLIP as a crucial factor regulating both the formation and the development of PC dendritic arbors during cerebellar development.

\section{Materials and Methods \\ Antibodies}

Primary antibodies used were as follows: purified rabbit polyclonal antiSCLIP (1:500) (Poulain and Sobel, 2007), monoclonal or rabbit polyclonal anti-28 kDa calbindin (CaBP) (1:5000; Swant), goat polyclonal anti-calbindin (CaBP D28K) (1:500; Santa Cruz Biotechnology), monoclonal (1:500; Roche) or polyclonal (1:500; Millipore) anti-green fluorescent protein (GFP), 9-1-E12 mouse monoclonal antibody against GAP-43 (1:5000; gift from Dr. J. H. Skene, Duke University, Durham, NC), monoclonal anti-parvalbumin (1:5000; Sigma-Aldrich), monoclonal anti-TGN38 (1:1000; Affinity BioReagents), monoclonal median Golgi marker CTR433 antibody (1:6; gift from Dr. M. Bornens, Institut Curie, Paris, France), anti-zebrin II (1:500; gift from Dr. R. Hawkes, University of Calgary, Calgary, Alberta, Canada), monoclonal antitubulin (1:10,000; Millipore), polyclonal anti-myc-tag (1:500; Cell Signaling Technology), Alexa 488-, 546-, and 633-conjugated secondary antibodies (1:1000; Invitrogen), and IRDye 800 - or Alexa 688conjugated secondary antibodies (1:5000; Rockland).

\section{Cryostat sections and immunocytochemistry}

Anesthetized rats were perfused intracardially with $4 \%$ paraformaldehyde in $0.12 \mathrm{M}$ phosphate buffer. Whole brains were postfixed overnight at $4^{\circ} \mathrm{C}$ in the same fixative, washed in PBS, and cryoprotected in PBS containing 30\% saccharose before being frozen in isopentane. Sagittal sections ( $14 \mu \mathrm{m}$ thick) were cut on a cryostat, delipidated with acetone at $-20^{\circ} \mathrm{C}$ for $10 \mathrm{~min}$, washed in PBS, permeabilized in PBS containing gelatin $(2 \mathrm{~g} / \mathrm{L})$ and $0.1 \%$ Triton X-100 for $2 \mathrm{~h}$, saturated with PBS containing $10 \%$ fetal calf serum, and incubated with primary antibodies overnight at $4^{\circ} \mathrm{C}$. After washing in PBS, sections were incubated with the appropriate secondary antibodies for $1 \mathrm{~h}$ at room temperature, washed in PBS, and mounted in mowiol (Calbiochem).

\section{Recombinant lentiviral production}

Plasmid constructions. The lentiviral construct (pFlap) (kindly provided by Dr. P. Charneau, CNRS, UMR7091, Paris, France) carries the DNA flap sequence (Zennou et al., 2001) and is deleted for the human immunodeficiency virus-1 (HIV-1) enhancer and promoter sequences in the U3 region of the 3' long-terminal repeat (Sirven et al., 2001). It also carries a GFP marker gene under the control of the phosphoglycerate kinase (PGK) promoter followed by a 600 bp posttranslational regulatory element, woodchuck posttranscriptional regulatory element (WPRE) (Zufferey et al., 1999). To inhibit SCLIP expression, a short hairpin RNA (shRNA) directed against SCLIP [described previously by Poulain and Sobel (2007)] was inserted into the pFlap-shRNA-PGK-GFP-WPRE vector and placed under the control of the human $\mathrm{U} 6$ promoter (LvshSCLIP). The corresponding control transfer vector (Lv-shCtrl) contained an inefficient sequence against SCLIP [previously described by Poulain and Sobel (2007)] in which three mismatches were further introduced. We checked the absence of match with any other coding or noncoding sequence in the whole genome database. To induce SCLIP overexpression, the mouse SCLIP cDNA fragment fused to a myc-tag was inserted into the pFlap-shRNA-PGK-GFP-WPRE vector deleted from its shRNA sequence and placed under the control of the PGK promoter in place of GFP (Lv-SCLIP). The same vector containing GFP alone was used as a control (Lv-GFP).

Lentiviral production. Stocks of viral particles were prepared as described previously (Naldini et al., 1996; Zennou et al., 2001) by transient cotransfection of HEK 293T cells with the p8.91 encapsidation plasmid (Zufferey et al., 1997), the pHCMV-G (vesicular stomatitis virus pseudotype) envelope plasmid and the pFlap vectors described above (Lv-shSCLIP, Lv-shCtrl, Lv-SCLIP, or Lv-GFP). Briefly, the supernatants were collected $48 \mathrm{~h}$ after transfection, treated with DNase (Roche) before ultracentrifugation, and then resuspended in PBS, aliquoted, and stored at $-80^{\circ} \mathrm{C}$ until use. The amount of $\mathrm{p} 24$ capsid protein was determined by the HIV-1 p24 ELISA antigen assay (Beckman Coulter). Virus from different productions averaged $150 \mathrm{ng} / \mu \mathrm{l}$ p 24 antigen. In addition, the transducing unit (TU) titers of the different lentiviral vector stocks were determined by transducing HEK 293T cells in 24-well plates with serial dilutions $\left(5 \mu \mathrm{l}, 5 \times 10^{-1} \mu \mathrm{l}, 5 \times 10^{-2} \mu \mathrm{l}, 5 \times 10^{-3} \mu \mathrm{l}, 5 \times 10^{-4} \mu \mathrm{l}\right.$, and $\left.5 \times 10^{-5} \mu \mathrm{l}\right)$. Cells were harvested by centrifugation and resuspended in a fixative solution of $1 \%$ paraformaldehyde $72 \mathrm{~h}$ after transduction. The number of GFP-positive cells was measured in a FACScan flow cytometer (BD Biosciences).

\section{Cerebellar slice cultures and infection}

Rats (OFA; Charles River) at embryonic day 19 (E19) or postnatal day 0 (P0) (date of birth) were used. Fetuses were obtained by cesarean section from pregnant rats deeply anesthetized with a lethal injection of sodium pentobarbital (200 mg/kg, i.p.). Briefly, after decapitation, brains of E19 or P0 rats were dissected out into cold Gey's balanced salt solution (Sigma-Aldrich) supplemented with $5 \mathrm{mg} / \mathrm{ml}$ glucose, and the meninges were removed. Parasagittal cerebellar slices ( $400 \mu \mathrm{m}$ thick) were cut on a Mcllwain tissue chopper and transferred onto $30 \mathrm{~mm}$ Millipore membrane culture inserts with $0.4 \mu \mathrm{m}$ pore size (Millicell CM; Millipore). The preparations were then infected by adding directly on the slices the volume of viral stock (Lv-shSCLIP, Lv-shCtrl, Lv-SCLIP, or Lv-GFP) corresponding to $\sim 1.5 \times 10^{4} \mathrm{TU}$. To study the effect of SCLIP inhibition at later phases of dendritic differentiation, some slices were infected $7 \mathrm{~d}$ after plating. Slices were maintained in culture in six-well plates containing $1 \mathrm{ml} /$ well medium made of $50 \%$ basal medium with Earle's salts (BME) (Invitrogen), 25\% HBSS (Invitrogen), 25\% horse serum, $1 \mathrm{~mm}$ L-glutamine, and $5 \mathrm{mg} / \mathrm{ml}$ glucose, at $37^{\circ} \mathrm{C}$ with $5 \% \mathrm{CO}_{2}$ for 7 or $14 \mathrm{~d}$, with fresh medium change every $2-3 \mathrm{~d}$.

\section{Cerebellar slice extracts and Western blot analysis}

For each time and infection condition, 10 cerebellar slices were taken from their membrane culture inserts, washed in cold PBS, lysed with $200 \mu \mathrm{l}$ of 10 mM Tris, pH 7.5, containing 2 mM EDTA, 1\% SDS, and protease inhibitor mixture (Roche Diagnostics), sonicated, and centrifuged at 20,000 $\times g$ for 15 $\min$ at $4^{\circ} \mathrm{C}$. Supernatants were then incubated with $1 \mathrm{ml}$ of acetone and centrifuged at $20,000 \times g$ for $15 \mathrm{~min}$ at $4^{\circ} \mathrm{C}$. Precipitated proteins were suspended and denatured in NuPAGE LSD sample buffer (Invitrogen), and then resolved on 12\% NuPAGE Bis-Tris gels (Invitrogen). They were transferred to $0.45 \mu \mathrm{m}$ nitrocellulose membranes in $50 \mathrm{~mm}$ Tris- $\mathrm{HCl}, \mathrm{pH} 7.4,40$ mm glycine, and 20\% isopropyl alcohol. Membranes were blocked with 5\% dry milk in PBS and incubated with primary antibodies in the same buffer. Primary antibodies were finally detected using IRDye 800- or Alexa 688conjugated secondary antibodies, which were visualized using the Odyssey Imaging System (LI-COR Biosciences). 

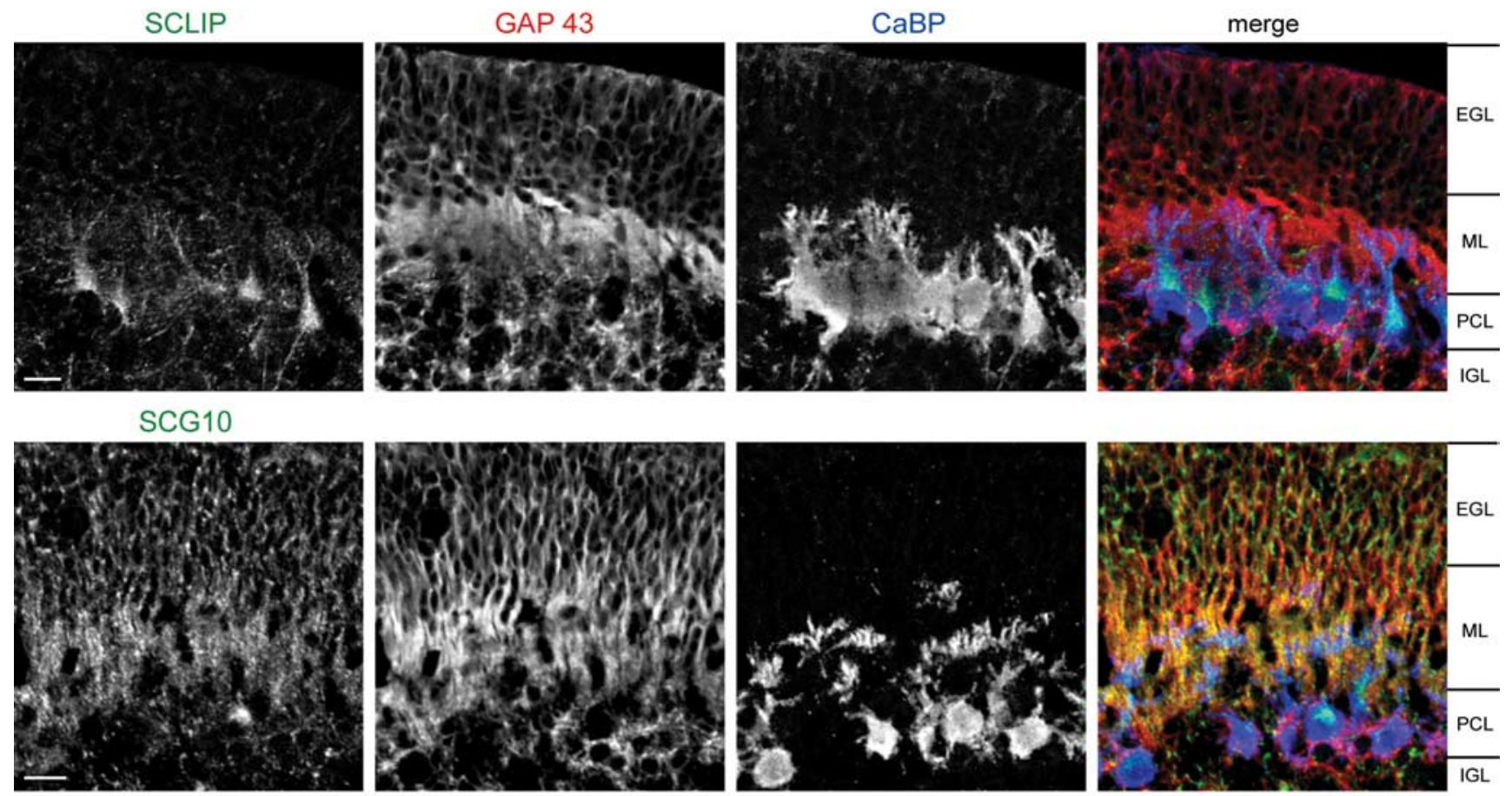

Figure 1. SCLIP and SCG10 have different distributions in the developing cerebellum. P10 rat cerebellar slices were immunostained for SCLIP or SCG10 (green), GAP-43 (red), and CaBP (specific marker for P(S; blue). SCLIP is strongly detected in PCs labeled by CaBP, whereas SCG10 is more particularly localized along the GAP-43-positive parallel fibers in the ML. Cerebellar slices from the hemispheric region of the cerebellum were used for a better visualization of the parallel fibers. Scale bar, $20 \mu \mathrm{m}$ (confocal microscopy; z-stack projection).

\section{Cerebellar slice immunostaining}

Cerebellar slices were fixed in 4\% paraformaldehyde in PBS for $1 \mathrm{~h}$ at room temperature, washed in PBS, and incubated for $1 \mathrm{~h}$ in PBS containing $0.25 \%$ Triton $\mathrm{X}-100,0.1 \mathrm{~m}$ gelatin, and $0.1 \%$ sodium azide. Primary antibodies used with appropriate dilutions were applied overnight. After washing in PBS, slices were incubated with secondary antibodies for $1 \mathrm{~h}$ at room temperature, washed in PBS, and mounted in mowiol.

\section{Imaging, quantification of PC differentiation stages, and} morphological measurements

Fluorescence microscopic images were obtained with a microscope (Leica Microsystems) equipped with a digital camera (Princeton Scientific Instruments), or with an SP2 confocal microscope (Leica).

Classification of PCs was assessed after CaBP immunostaining among the transduced PCs identified by GFP. We classified PC differentiation stages as described previously (Boukhtouche et al., 2006). Briefly, fusiform PCs with a bipolar shape were defined as stage 1, stellate PCs with processes all around the soma as stage 2, PCs with several long and mature perisomatic dendritic processes as stage 3 , and PCs with a single dendritic tree giving rise to additional side branches as stage 4 . It should be noted that stage 3 with the presence of more than one primary dendritic tree is specific of organotypic cultures (Dusart et al., 1997; Kapfhammer, 2004) and appears in parallel with stage 4 . For each experiment, at least 100 transduced PCs from all slices were quantified. Results are expressed as means \pm SEM ( $n=3$ or 4 independent experiments). The statistical significance of differences between repartitions obtained in Figure 6 was assessed by a $\chi^{2}$ independence test.

Primary dendrites were defined as dendritic processes emerging from PC somas. Primary branches were counted for all dendritic processes of transduced PCs at stage 3. Mean values were calculated from three independent experiments and compared with a Mann-Whitney two-tailed test using Prism software.

\section{Results}

SCLIP is highly expressed in Purkinje cells and accumulates in their growing dendrites during cerebellar development Previous in situ hybridization experiments revealed a strong expression of SCLIP mRNA in adult rat PCs, in contrast to the weakly detected other stathmin family members SCG10 and stathmin (Ozon et al., 1999). To assess whether SCLIP was also specifically expressed in PCs during development and at the protein level, we used specific immunolabeling to compare the cellular expression of SCLIP and SCG10 proteins in the developing cerebellum (Fig. 1). Both proteins were detected in the various cerebellar layers at P10, but exhibited different distributions: SCLIP immunoreactivity was essentially confined to the Purkinje cell layer (PCL), as evidenced by colabeling with $\mathrm{CaBP}$, a widely used PC marker, whereas SCG10 was predominantly observed in the molecular layer $(\mathrm{ML})$, where it colocalized with the parallel fibers marker GAP-43 (Wehrlé et al., 2001), and in the external granular layer (EGL). The specific enrichment of SCLIP in the PCL prompted us to characterize more precisely its expression and localization throughout postnatal rat cerebellar development (Fig. 2A,B). Immunolabeling at different ages (P3, P7, $\mathrm{P} 10$, and P15) revealed that SCLIP is highly expressed in PCs until postnatal day 10 , after which it decreases significantly (Fig. $2 A$ ). It was also detected as a punctuate labeling in the EGL and internal granular layer (IGL) (Fig. 2A).

PCs undergo substantial morphological changes during the first 15 postnatal days in vivo in rats (Armengol and Sotelo, 1991), from a bipolar morphology at the end of their migration to a more complex stage corresponding to the elaboration of their mature dendritic tree. At P3, when some PCs have a bipolar morphology, SCLIP immunoreactivity was observed in their soma, concentrated in the perinuclear Golgi region at the starting point of the dendrite (Fig. 2 B, P3, arrowheads). At P7 (Fig. 2, P7), when PCs exhibit several protruding disoriented short dendrites devoid of spines, SCLIP was still detected in the perinuclear Golgi region (arrowheads) as well as in some dendritic protrusions extending in the ML (arrow). At P10, most PCs have a single main dendrite leaving the cell body, with only few dendritic branches. Interestingly, SCLIP immunoreactivity was very strong in these developing dendrites and branches, with punctuate SCLIP stain- 

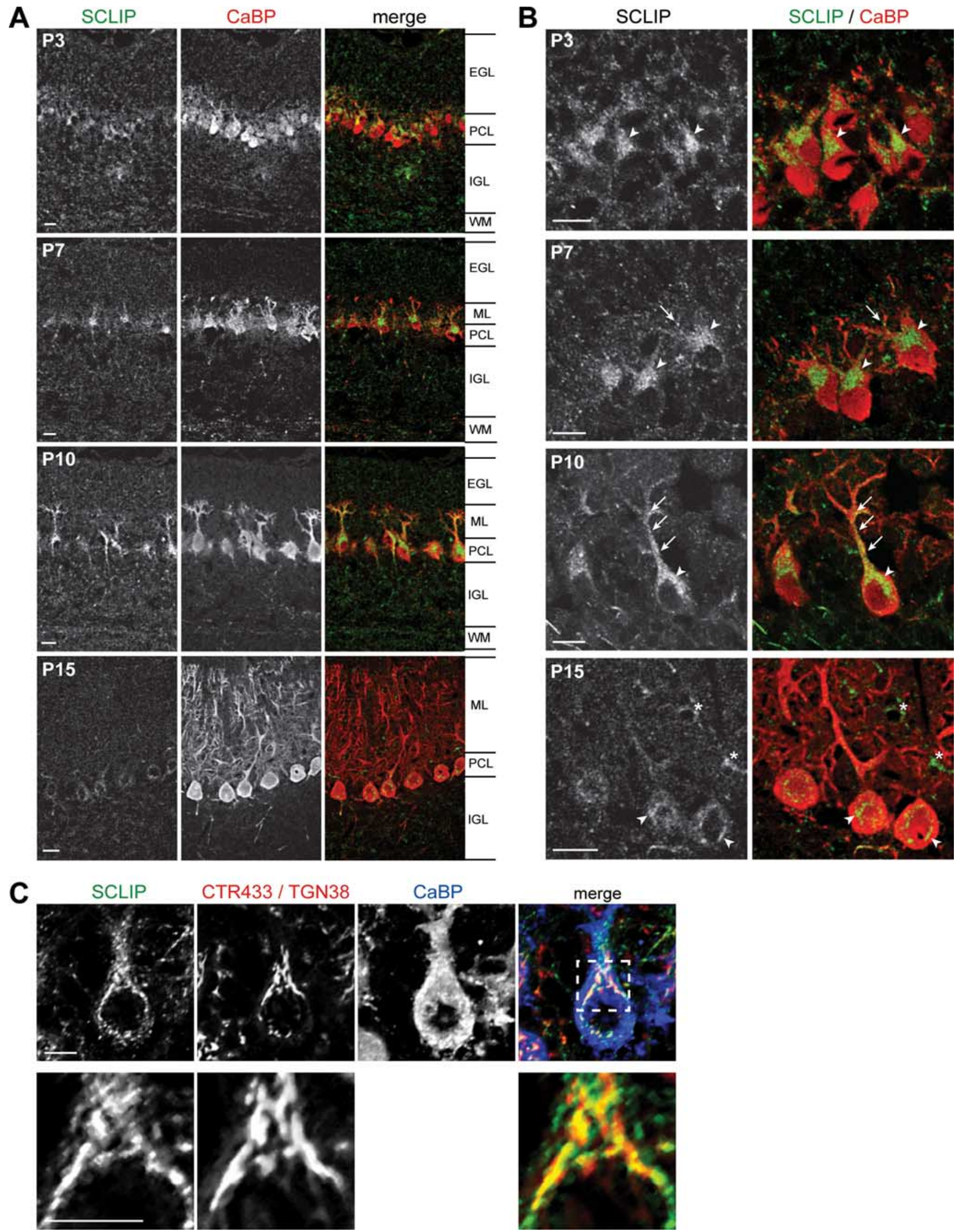

Figure 2. SCLIP is highly expressed in PCs and targeted to their growing dendrites during cerebellar development. $A, B$, Rat cerebellar slices were immunostained for SCLIP (green) and CaBP (red) at different ages of development and observed at low $(\boldsymbol{A})$ and high $(\boldsymbol{B})$ magnification. From P3 to P10, SCLIP is present in the different cerebellar layers but is predominantly detected in the PCL, where it appears localized in the perinuclear Golgi region (arrowheads) and developing dendrites (arrows) of PCS. At P10, SCLIP is more particularly accumulated in the principal trunk and secondary branches of PC dendritic arbors (arrows) in addition to the perinuclear Golgi region (arrowhead). At P15, however, SCLIP labeling is strongly reduced and only weakly detectable in PC somas (arrowheads). The asterisks at P15 indicate interneurons in the molecular layer also displaying SCLIP labeling in their soma [supplemental Fig. 1, available (Figure legend continues.) 
A

a

$b$
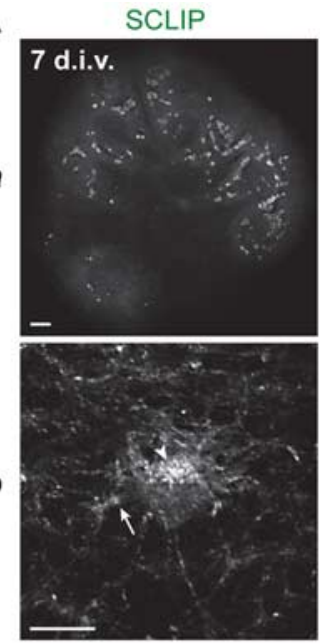

B

a

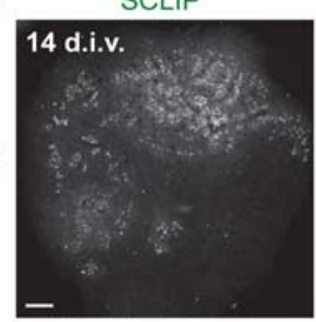

$b$

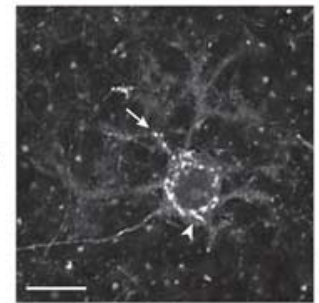

$c$

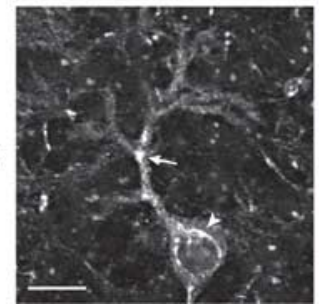

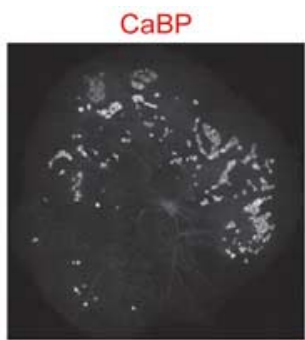
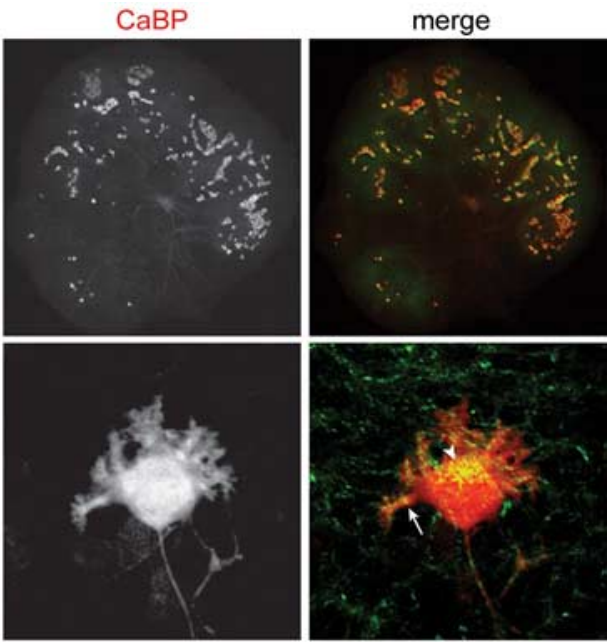

CaBP
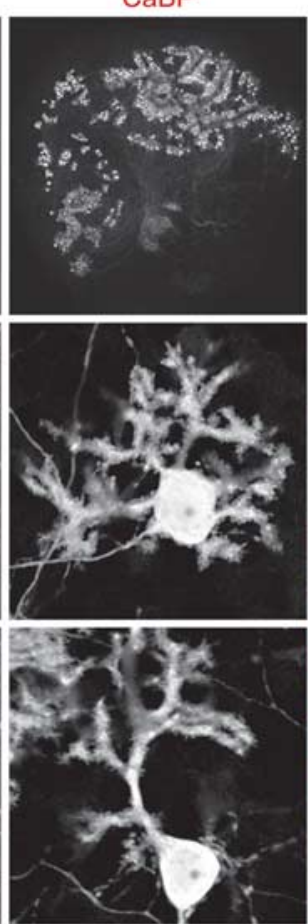
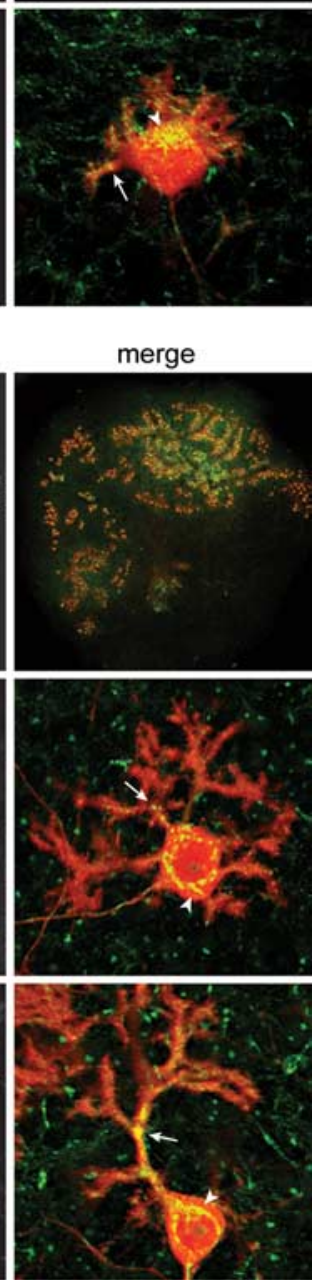

Figure 3. SCLIP is highly expressed in PCs in cerebellar organotypic cultures. Cerebellar organotypic cultures were immunostained for SCLIP (green) and CaBP (red) after $7(\boldsymbol{A})$ and $14(\boldsymbol{B})$ div. As can be seen at low magnification ( $\boldsymbol{A} \boldsymbol{a}, \boldsymbol{B} \boldsymbol{a})$, SCLIP labeling follows the general pattern of $\mathrm{PC}$ s at both 7 and 14 div, indicating that $\mathrm{PC}$ s express most SCLIP in cerebellar slices cultured in vitro. Higher magnifications $(\boldsymbol{A} \boldsymbol{b} ; \boldsymbol{B} \boldsymbol{b}, \boldsymbol{c})$ further show SCLIP immunoreactivity in the perinuclear Golgi region (arrowheads) and dendritic processes (arrows) of PCs at the various typical developmental stages, from stage 2 to stage 4 (Fig. $5 \mathrm{~A}$ ). Scale bars: $200 \mu \mathrm{m}$ (lower magnifications); $20 \mu \mathrm{m}$ (higher magnifications; confocal microscopy).

ing in the principal trunk and secondary branches (Fig. $2 \mathrm{~B}, \mathrm{P} 10$, arrows). SCLIP was also detected in the soma (Fig. $2 B$, P10, arrowhead), partially at the Golgi complex as evidenced by colabeling with CTR433 and TGN38 antibodies (Fig. 2C). In contrast, at P15, when PCs display a mature elaborate dendritic arbor with

(Figure legend continued.) at www.jneurosci.org as supplemental material]. WM, White matter. Scale bar, $20 \mu \mathrm{m}$ (confocal microscopy). C, P10 rat cerebellar slices were immunostained for SCLIP (green), CTR433 and TGN38 (specific markers for median- and trans-Golgi; red), and CaBP (blue). SCLIP appears in the soma as a punctuate labeling in part at the CTR433- and TGN38labeled Golgi complex, as shown in more detail at higher magnification (bottom). Scale bar, 10 $\mu \mathrm{m}$ (confocal microscopy; z-stack projection).

numerous branches, SCLIP labeling appeared strongly decreased (Fig. 2A, P15). It was no longer observed in the differentiated dendrites and became only weakly detectable in the perinuclear Golgi region (Fig. 2B, P15, arrowheads). A similarly punctuate labeling was also present in the soma and processes of interneurons in the ML (Fig. 2B, P15, asterisks; supplemental Fig. 1, available at www.jneurosci.org as supplemental material).

SCLIP thus appears as a highly expressed protein in differentiating PCs during cerebellar development. Its accumulation in dendritic processes at a critical period of dendrite formation and outgrowth in vivo further suggested a specific role in PC dendritogenesis.

\section{SCLIP is strongly expressed in developing Purkinje cells in organotypic culture}

We therefore investigated the functions of SCLIP in PC dendritic development in rat, using cerebellar organotypic cultures. Such cultures offer the great advantage of maintaining structural and physiological conditions that allow a dendritic development of PCs almost similar to that observed in vivo, albeit in a somewhat delayed manner (Fig. 3) (see Fig. 5A) (Kapfhammer, 2004). We first observed that, as previously described in the mouse (Boukhtouche et al., 2006), rat PCs in organotypic slices cultured at $\mathrm{P} 0$ are bipolar and fusiform (stage 1 ) during the very first days in vitro (div). This stage corresponds to the early fusiform stage described in vivo (Ramón y Cajal, 1926; Armengol and Sotelo, 1991). After 7 div, most PCs have retracted their primitive processes and developed numerous perisomatic protrusions (Fig. $3 A b$, stage 2). This stage corresponds to the stellate stage with disoriented dendrites observed in vivo (Ramón y Cajal, 1926; Armengol and Sotelo, 1991). After 14 div, beside stage 2, two other PC morphologies appeared concomitantly: stage 3 , with multipolar dendritic processes (Fig. $3 B b$ ), and stage 4 , with a single main dendrite (Fig. $3 B c$ ). Numerous branches and spines were detected at these two stages, which correspond to mature PCs and occur in parallel. In vivo, only stage 4 is observed, the presence of stage 3 cells with multipolar primary dendritic processes being attributable to the organotypic culture conditions (Dusart et al., 1997; Kapfhammer, 2004).

We then characterized the expression and localization of SCLIP in the PCs of rat organotypic cultures (Fig. 3). Similar to our in vivo observations, after 7 and 14 div, SCLIP was predominantly detected in PCs, as shown by colabeling with CaBP (Fig. $3 A a, B a)$. At stage 2 after 7 div, SCLIP appeared accumulated in the perinuclear Golgi region of PCs (arrowhead) and was also detected in their short perisomatic protrusions (Fig. $3 A b$, arrow). 
Such a localization was still observed after 14 div (Fig. 3B), when SCLIP was present in the soma (arrowhead) and main dendrites (arrow) of PCs at stages 3 (Fig. $3 B b$ ) and 4 (Fig. $3 B c$ ).

SCLIP thus displayed a similar expression and localization in PCs of rat cerebellar organotypic cultures as observed in vivo, confirming that this model is appropriate to investigate its specific roles in PC dendritogenesis.

\section{Lentiviral-mediated RNA interference in cerebellar organotypic cultures inhibits SCLIP expression in Purkinje cells}

To investigate the specific functions of SCLIP in PC dendritic differentiation, we developed a lentiviral-mediated RNA interference (RNAi) approach to inhibit SCLIP expression in PCs in cultured rat cerebellar slices. We produced a recombinant nonreplicative lentiviral vector encoding a small hairpin RNA inhibiting efficiently the expression of SCLIP (Poulain and Sobel, 2007) and GFP as a transduction marker (Lv-shSCLIP). The same lentiviral vector encoding an inefficient sequence against SCLIP (Poulain and Sobel, 2007) in which three mismatches were further inserted was used as a control (Lv-shCtrl). We first evaluated the ability of these vectors to efficiently transduce PCs in cerebellar organotypic cultures. As shown on Figure $4 A$ for Lv-shCtrl, P0 cerebellar slices infected just after plating displayed after $7 \mathrm{~d}$ (data not shown) and $14 \mathrm{~d}$ (Fig. 4A) in culture numerous transduced cells visualized by GFP, a large proportion of which were identified as PCs by colabeling with CaBP. The lentiviral vector approach thus appeared to efficiently transduce PCs in cerebellar organotypic cultures.

We then assessed the efficacy of Lv-shSCLIP for inhibiting SCLIP expression in PCs by immunocytochemical analyses. In contrast to the $\mathrm{Lv}$-shCtrl infected slices in which all $\mathrm{PCs}(\mathrm{CaBP}+)$ expressed SCLIP (Fig. 4B), very few SCLIP-positive cells were observed in the Lv-shSCLIP-infected slices (Fig. 4B, arrowheads), in which numerous Purkinje cells were transduced $(\mathrm{GFP}+/ \mathrm{CaBP}+)$. As evidenced at higher magnification, SCLIP labeling was indeed dramatically reduced at both 7 and 14 div in the Lv-shSCLIP transduced PCs (Fig. 4C, GFP+/CaBP+, arrows), whereas it remained unchanged in untransduced PCs (Fig. $4 C$, GFP-/CaBP +, arrowheads) or in PCs transduced with LvshCtrl (supplemental Fig. 2, GFP+/CaBP+, arrowheads; available at www.jneurosci.org as supplemental material).

This observation was further validated by SCLIP and GFP Western blot analyses of slice cultures infected with Lv-shSCLIP after 1 and 7 div. The expression of SCLIP strongly decreased during the first $7 \mathrm{~d}$ in culture, whereas that of GFP increased and the levels of the control proteins tubulin and zebrin II, a PCspecific protein (Brochu et al., 1990), were not affected during this period (Fig. $4 D$ ).

Together, both immunocytochemical and Western blot analyses clearly demonstrate that the Lv-shSCLIP vector is highly efficient to inhibit SCLIP expression in developing PCs in cerebellar organotypic cultures.

\section{SCLIP inhibition prevents PC dendrite formation and development}

To investigate the effects of SCLIP knock-down on postnatal PC dendritic development, we infected P0 cerebellar organotypic cultures with Lv-shSCLIP or Lv-shCtrl just after plating and analyzed PC morphology and differentiation stages (Fig. $5 A$ ) at 7 and $14 \mathrm{div}$ (Fig. 5B-D).

After 7 div (Fig. $5 B, C$ ), the majority $[89.5 \pm 3.0 \%$ (SEM) $]$ of PCs transduced with Lv-shCtrl $(\mathrm{GFP}+/ \mathrm{CaBP}+)$ were in stage 2 , displaying short and dense perisomatic protrusions, like untransduced PCs (GFP-/CaBP+). In contrast, almost all (98.5 土 $0.7 \%)$ PCs transduced with Lv-shSCLIP $(\mathrm{GFP}+/ \mathrm{CaBP}+)$ and hence lacking SCLIP displayed a "round" morphology, without any process emerging from their somas but the axon, which seemed not affected (arrowheads). Their neighboring untransduced PCs exhibited instead a typical stage 2 morphology, indicating that SCLIP knock-down in PCs was directly responsible for inducing the round shape. Thus, the lack of SCLIP during the first week in vitro prevents the formation of $\mathrm{PC}$ perisomatic protrusions during these first steps of PC dendritic development.

After 14 div (Fig. 5D,E), the effect of SCLIP depletion on PC dendritic development was even more pronounced. PCs transduced with Lv-shCtrl were like untransduced cells in stage 3 $(53.5 \pm 2.5 \%)$ or $4(34.8 \pm 1.4 \%)$ with several branched dendritic processes or typical single dendritic arbors, respectively. In contrast, PCs transduced with Lv-shSCLIP still exhibited a round morphology $(73.6 \pm 3.4 \%)$ (asterisks) or formed very short individual "finger-like" protrusions (26.4 $\pm 3.4 \%)$ (arrows). Remarkably, neighboring untransduced PCs in Lv-shSCLIP infected slices displayed normal stage 3 or stage 4 morphologies.

Thus, early inhibition of SCLIP expression during the first 2 weeks in vitro dramatically prevents postnatal PC dendritic development in cerebellar organotypic cultures, revealing a crucial role for SCLIP in the formation of PC dendritic arbors. This result prompted us to ask whether SCLIP also regulates the earlier phases of PC differentiation (i.e., retraction of the primitive stage 1 process) or the later phases (i.e., elongation and branching of the mature dendritic tree). We therefore inhibited SCLIP expression in PCs first at an embryonic, and then at a more mature stage.

\section{SCLIP inhibition promotes process retraction of stage 1 fusiform PCs}

SCLIP depletion prevents the formation of stage 2 PC perisomatic protrusions, leading to the absence of dendritic processes at both 7 and 14 div. These protrusions are normally formed after retraction of the primitive processes of stage 1 fusiform PCs. At E19 in vivo, when PCs are mostly in stage 1, SCLIP immunoreactivity was detected in the soma and along the primitive processes of most peripheral PCs (Fig. $6 \mathrm{~A}$, arrows), suggesting a likely role also in early PC dendritogenesis.

To investigate whether SCLIP inhibition might lead to retraction of the primitive processes of stage 1 fusiform PCs, we infected E19 cerebellar organotypic cultures with Lv-shSCLIP or Lv-shCtrl just after plating and analyzed PC morphology and differentiation stages at 6 and 7 div (Fig. 6C-E). Lv-shSCLIP transduction induced a complete inhibition of SCLIP expression in PCs after 6 div (Fig. 6B, arrow) and 7 div (data not shown) compared with untransduced PCs (Fig. $6 B$, arrowhead). After 6 (Fig. 6C,D) and 7 (Fig. 6E) div, many untransduced PCs (GFP-/ $\mathrm{CaBP}+)$ and PCs transduced with $\mathrm{Lv}$-shCtrl $(\mathrm{GFP}+/ \mathrm{CaBP}+)$ still exhibited a bipolar stage 1 morphology (6 div, $51.5 \pm 8.2 \%$; 7 div, $37.9 \pm 7.5 \%$ ) (arrowheads), most of the others being in a stellate stage 2 with perisomatic protrusions ( $6 \mathrm{div}, 38.0 \pm 6.7 \%$; 7 div, $55.5 \pm 3.4 \%$ ) (asterisk). In contrast, almost all (6 div, $88.3 \pm 4.4 \% ; 7$ div, $87.0 \pm 3.4 \%)$ PCs transduced with LvshSCLIP $(\mathrm{GFP}+/ \mathrm{CaBP}+)$ displayed a round morphology (Fig. $6 C)$ similar to that observed after infection of P0 organotypic cultures. Their neighboring untransduced PCs $(\mathrm{GFP}-/ \mathrm{CaBP}+)$ exhibited a typical bipolar morphology (Fig. 6C, arrow). Thus, the round shape was a direct consequence of SCLIP depletion, 
A

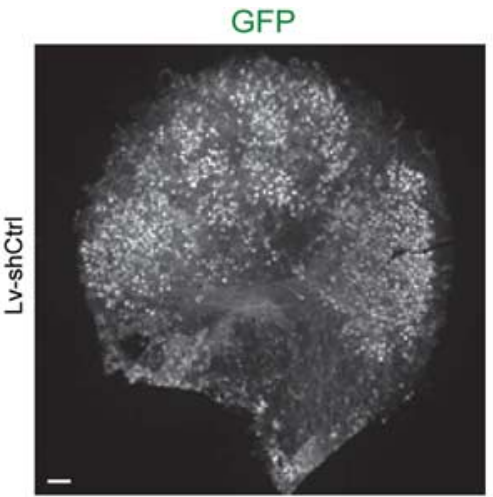

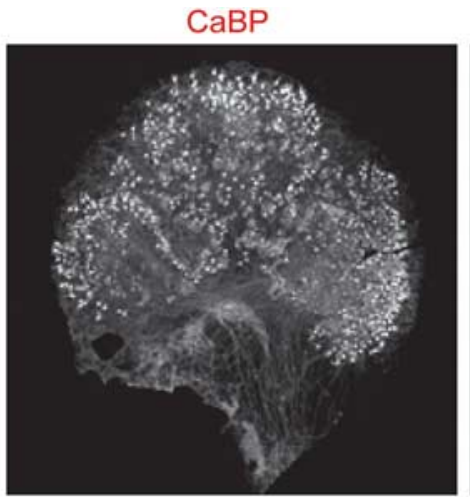

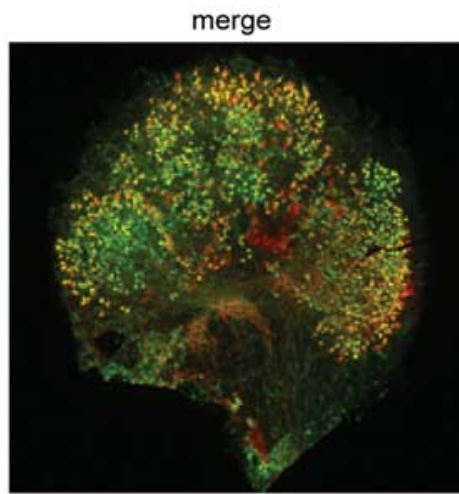

B
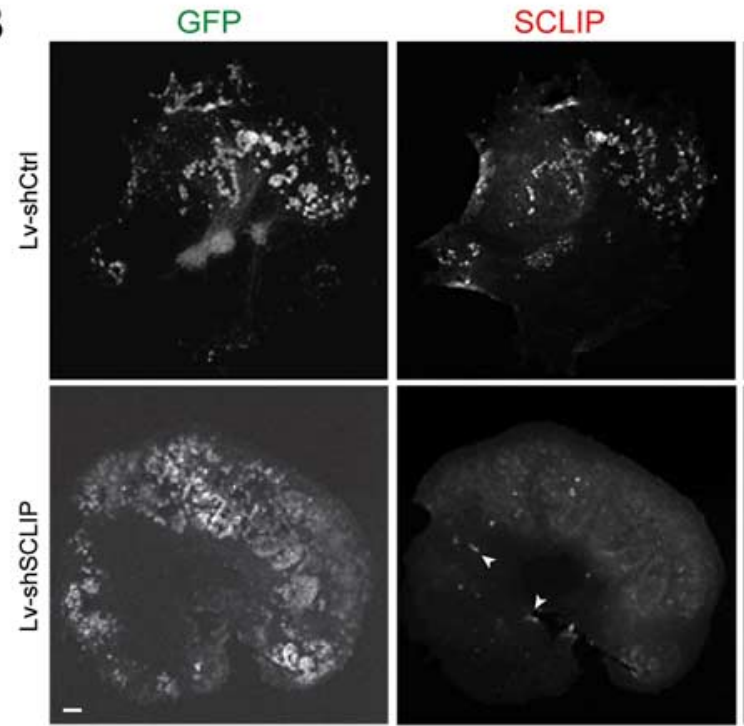

C
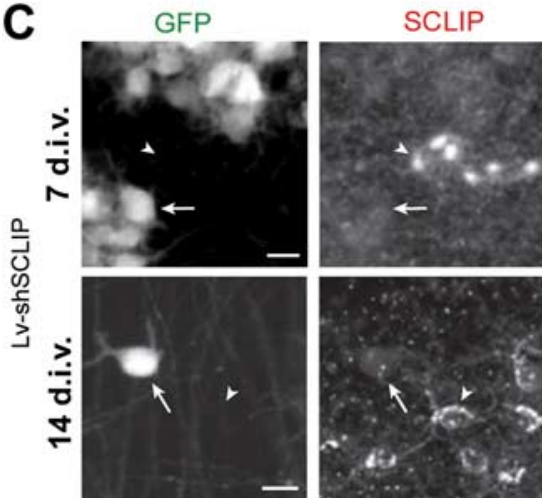

CaBP
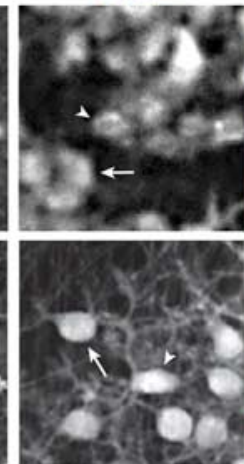
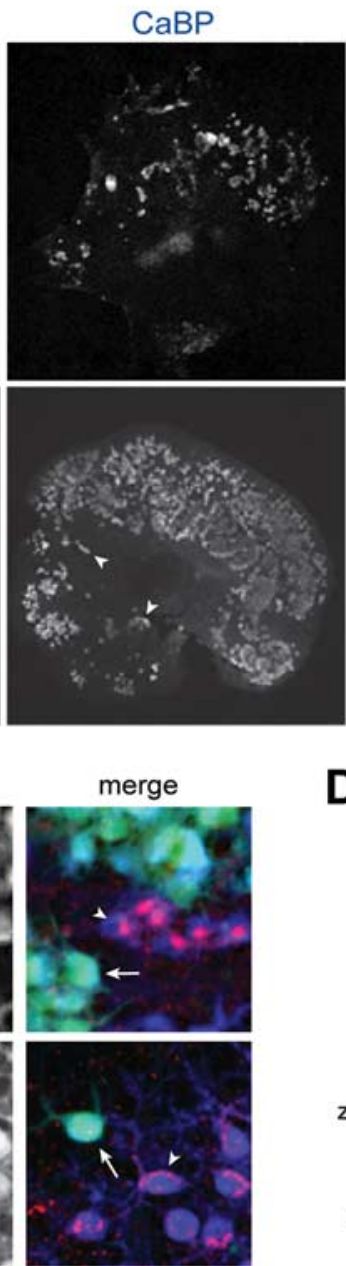

D

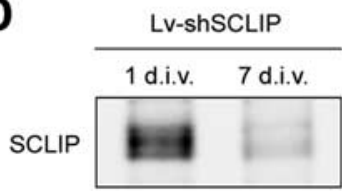

GFP

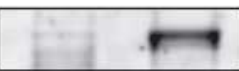

zebrin II

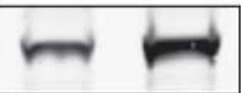

tubulin

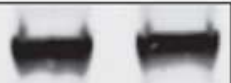

Figure 4. Lentiviral-mediated RNAi is adapted to study SCLIP function in PC dendritic development. $\boldsymbol{A}$, Lentiviral-mediated transduction in organotypic cultures allows gene transfer in PCS. Neonatal cerebellar organotypic cultures were infected with Lv-shCtrl just after plating and immunostained for GFP (green) and CaBP (red) $14 \mathrm{~d}$ later. Among transduced cells visualized by GFP, numerous cells were identified as PCs, as revealed by colabeling with CaBP. Scale bar, $200 \mu \mathrm{m}$. B, Lentiviral-mediated RNAi inhibits SCLIP expression in PCs. Cerebellar organotypic cultures were infected with the Lv-shCtrl or the Lv-shSCLIP vectors just after plating and immunostained for GFP (green), SCLIP (red), and CaBP (blue) $7 \mathrm{~d}$ later. SCLIP labeling is no more observed in PCs transduced with Lv-shSCLIP (visualized by GFP), whereas it remains clearly detected in untransduced PCs (arrowheads) or Lv-shCtrl transduced PCs (top panels). Scale bar, $200 \mu \mathrm{m}$. C, Higher magnifications showing the inhibition of SCLIP expression in PCs transduced by Lv-shSCLIP after 7 and 14 div. At both 7 and 14 div, SCLIP labeling is no more detected in transduced PCs visualized by GFP (arrows), whereas it remains high in untransduced PCS (arrowheads). Scale bar, $20 \mu \mathrm{m}$. D. Western blot characterization of Lv-shSCLIP efficiency for inhibiting SCLIP expression. Cerebellar organotypic cultures were infected with the Lv-shSCLIP vector just after plating and protein expression was evaluated 1 and $7 \mathrm{~d}$ later. SCLIP expression is strongly inhibited at 7 div, whereas that of GFP concomitantly increases. In contrast, tubulin or zebrin (specific of P(s) expression is not significantly affected by the lentiviral-mediated RNAi treatment.

resulting both from an accelerated primitive process retraction and inhibited secondary dendritic outgrowth.

SCLIP thus appears as a crucial regulator of the early stages of $\mathrm{PC}$ dendritogenesis, controlling both regression and growth processes.

\section{SCLIP inhibition blocks PC dendrite elongation and branching}

We then investigated whether SCLIP also plays a regulatory role during later phases of dendritic morphogenesis. SCLIP expression was inhibited in $\mathrm{P} 0$ cerebellar organotypic cultures by infec- 

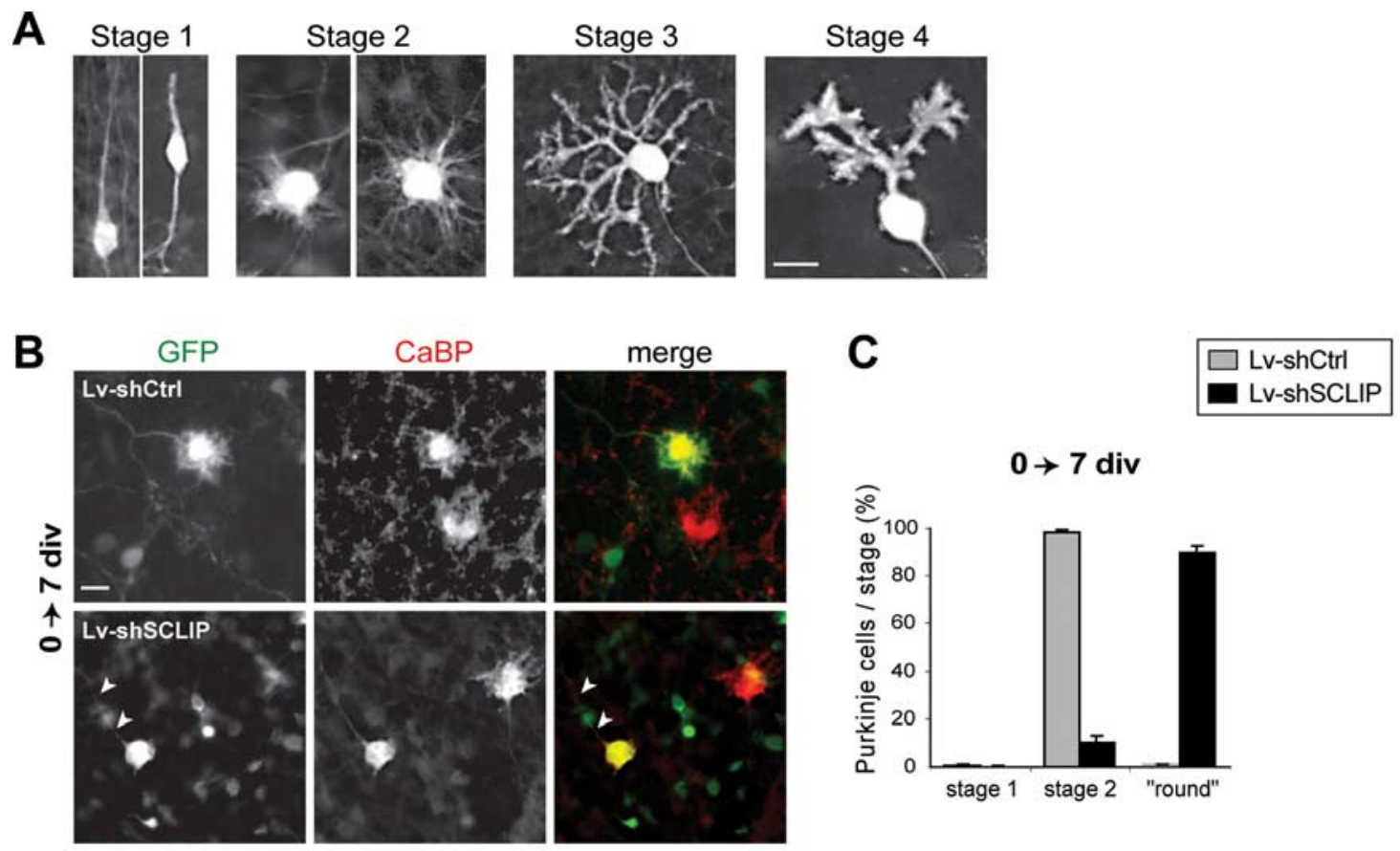

D

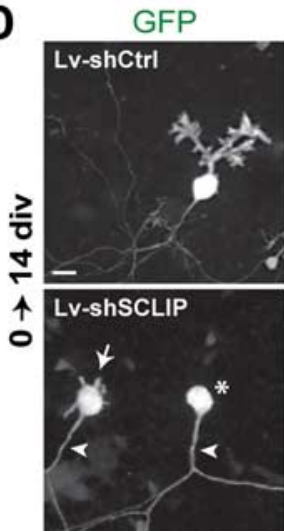

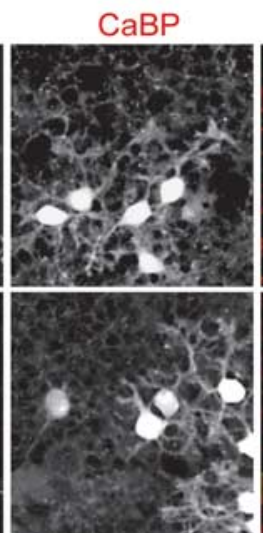

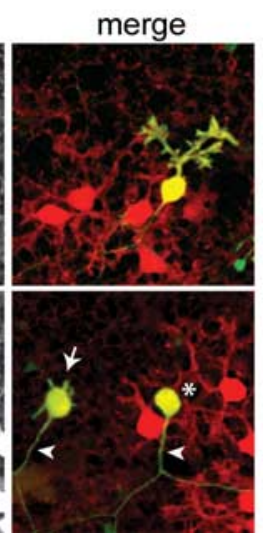

$\mathbf{E}$

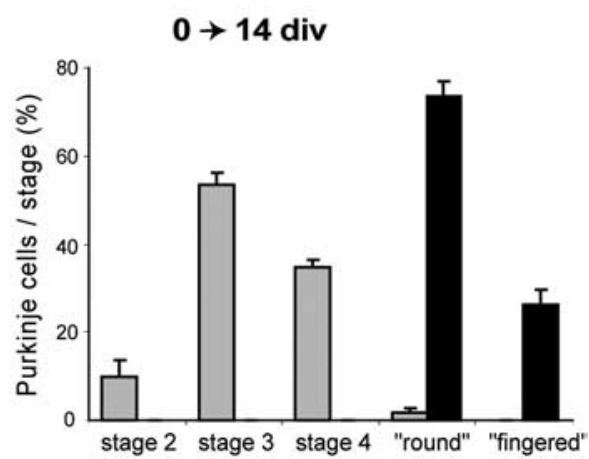

Figure 5. Early SCLIP inhibition blocks $P C$ dendritic development at 7 and 14 div. $A$, Classification of $P C$ differentiation stages in cerebellar organotypic cultures. Rat cerebellar slices were immunostained for CaBP after $3 \mathrm{~d}$ (stage 1), $7 \mathrm{~d}$ (stage 2), and $14 \mathrm{~d}$ (stages 3 and 4) in culture. P(s first display a fusiform bipolar morphology referred to as stage 1, and then exhibit short perisomatic processes characteristic of stellate stage 2. They finally display several perisomatic dendritic processes and eventually a single typical dendritic tree, respectively defined as stages 3 and 4 . Scale bar, $20 \mu \mathrm{m}$. B , Morphologies of PCs at 7 div after early infection with Lv-shCtrl or Lv-shSCLIP. Cerebellar organotypic cultures were infected just after plating and immunostained for GFP ( $g$ reen) and CaBP (red) 7 d later. Whereas PCs transduced with Lv-shCtrl (visualized by GFP) are in stage 2 and display short perisomatic protrusions as do untransduced PCs (red), PCs transduced with Lv-shSCLIP exhibit a round morphology, without any perisomatic processes but the axon (arrowheads). Scale bar, $20 \mu \mathrm{m}$. C, Quantified results of four combined independent experiments illustrating the effect of SCLIP inhibition on PC dendritic differentiation stages at 7 div. Whereas almost all PCs transduced with Lv-shCtrl are in stage $2,>90 \%$ of PCs transduced with Lv-shSCLIP are in a round stage. Shown are mean values \pm SEM. D, Morphologies of PCs at 14 div after early infection with Lv-shCtrl or Lv-shSCLIP. Cerebellar organotypic cultures were infected just after plating and immunostained for GFP (green) and CaBP (red) 14 d later. In contrast to untransduced PCs or PCs transduced with Lv-shCtrl, which exhibit a large and branched dendritic arbor, PCs transduced with Lv-shSCLIP still display a round morphology (asterisks) or exhibit very short fingered processes (arrows) emerging from the cell body. Note that their axons are, however, not affected by SCLIP inhibition (arrowheads). Scale bar, $20 \mu \mathrm{m}$. E, Quantified results of four combined independent experiments illustrating the effects of SCLIP inhibition on PC dendritic differentiation stages at 14 div. Whereas most of PCs transduced with Lv-shCtrl are in stages 3 and $4, \sim 75$ and $25 \%$ of PCs transduced with Lv-shSCLIP are in a round or "digitated" stage, respectively. Values shown are mean \pm SEM.

tion with Lv-shSCLIP at 7 div, when most PCs have reached stage 2 , instead of immediately after plating. We then examined PC morphology and differentiation stages $7 \mathrm{~d}$ after infection, at 14 div, in comparison with cultures infected similarly with Lv-shCtrl (Fig. 7).

After late infection, although the number of transduced PCs was lower than at earlier infection (data not shown), PCs transduced with Lv-shCtrl still presented several dendritic arborizations (stage 3 ) or typical dendritic trees with numerous branches and spines (stage 4) (data not shown). In contrast, most PCs transduced with Lv-shSCLIP displayed abnormal morphologies that could be classified into four types. The round and "fingered" morphologies (described previously) displayed no dendritic process, whereas the two other types displayed several (Fig. 7Aa) or one (Fig. $7 A b$ ) dendrite(s), which appeared thicker and mostly devoid of branches or spines, and occasionally presented a forked extremity. We refer to PCs with more than one dendrite as "branchless stage 3" (Fig. 7Aa) and to those with only one dendrite as "branchless stage 4" (Fig. 7Ab). Whereas most control PCs were in stage $3(53.1 \pm 11.1 \%)$ or $4(41.5 \pm 10.7 \%)$, SCLIP depleted PCs were mostly in a branchless stage $3(34.5 \pm 4.0 \%)$ or branchless stage $4(28.3 \pm 5.8 \%)$ morphology (Fig. $7 B)$. Almost all other PCs lacking SCLIP were in a round or fingered stage $(36.2 \pm 6.7 \%)$. 
A
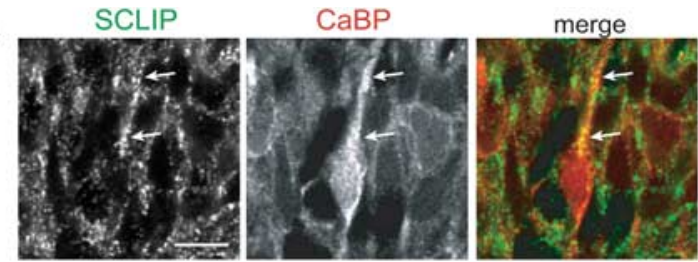

B
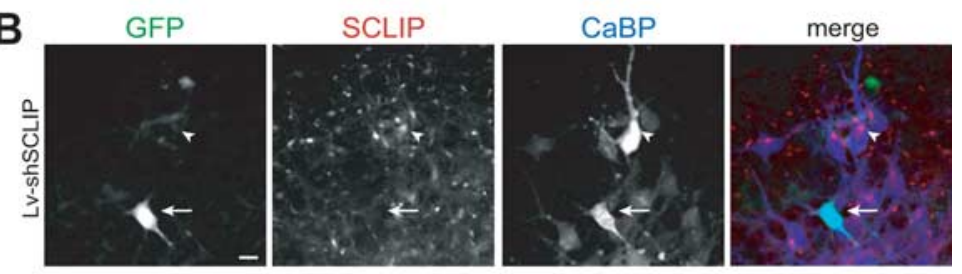

C
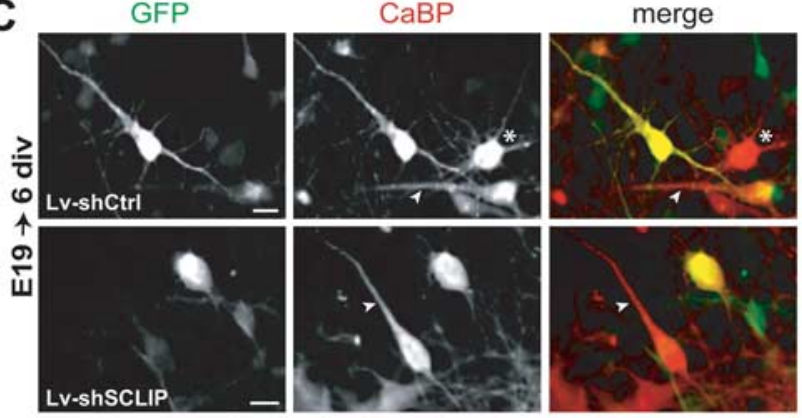

D

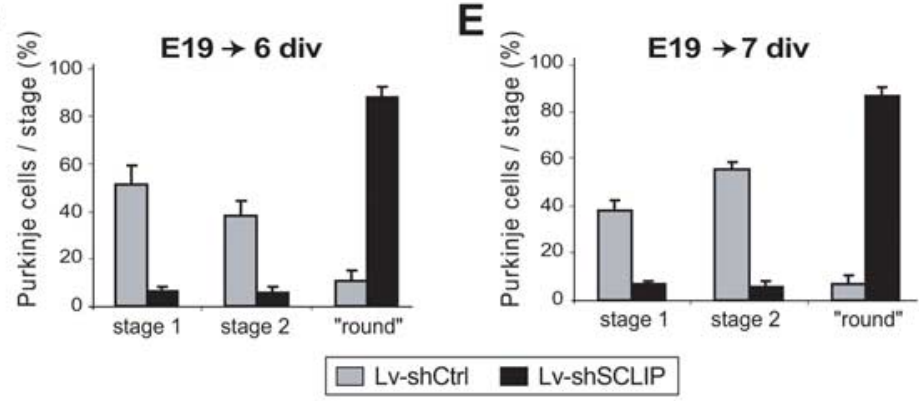

Figure 6. SCLIP inhibition at early stages promotes primitive process retraction of stage 1 fusiform $\mathrm{PCS}$. $A, P C$ morphology observed in vivo at E19. E19 rat cerebellar slices were immunostained for SCLIP (green) and CaBP (red). At E19, many PCs have reached their final destination in the developing cerebellum but still exhibit a bipolar morphology with a single primitive process (arrows) beside the axon. SCLIP is present in PCs and is particularly detected as a punctuate staining along the main process (arrows). Scale bar, $20 \mu \mathrm{m}$ (confocal microscopy; z-stack projection). B, Lentiviral-mediated RNAi inhibits SCLIP expression in PCs of 19 cerebellar cultures. E19 cerebellar organotypic cultures were infected with Lv-shSCLIP just after plating and immunostained for GFP (green), SCLIP (red), and CaBP (blue) after 6 div. SCLIP labeling is no more detected in transduced PCs visualized by GFP (arrow), whereas it remains clearly visible in untransduced PCs (arrowhead). Scale bar, $20 \mu \mathrm{m}$. C, Morphologies of PCs at 6 div after infection of E19 cerebellar cultures with Lv-shCtrl or Lv-shSCLIP. E19 cerebellar organotypic cultures were infected just after plating and immunostained for GFP (green) and CaBP (red) after 6 div. Whereas PCs transduced with Lv-shCtrl (visualized by GFP) are mostly in stage 1 with a bipolar morphology like untransduced PCs (red; arrowheads), PCs transduced with Lv-shSCLIP exhibit a round morphology without any process beside the thin axon. The asterisk indicates the morphology of a stellate stage 2 PC. Scale bar, $20 \mu \mathrm{m}$. D, E, Quantified results of three combined independent experiments illustrating the effect of SCLIP inhibition on PC dendritic differentiation stages at 6 and 7 div. Whereas PCs of E19 cerebellar cultures transduced with Lv-shCtrl are mostly in stages 1 or 2 after $6(\boldsymbol{D})$ and $7(\boldsymbol{E})$ div, respectively, $\sim 88 \%$ of PCs transduced with LV-shSCLIP are in a "round stage." Values shown are mean \pm SEM.

Together, these observations reveal that SCLIP inhibition prevents dendrite outgrowth and branching at later stages of PC dendritic development. SCLIP thus appears to tightly regulate late PC dendritic morphogenesis in addition to being crucial for early dendrite formation.

\section{SCLIP overexpression promotes PC dendritic development after 14 div}

The dramatic effects of SCLIP silencing on PC dendritogenesis prompted us to investigate conversely the consequences of SCLIP overexpression. We developed a recombinant lentiviral vector encoding SCLIP fused to a myc-tag (Lv-SCLIP) to trigger SCLIP expression in PCs. Because a strong expression of SCLIP is deleterious for neurite development (Poulain and Sobel, 2007), we used a plasmidic configuration such that SCLIP-myc expression was weaker and delayed. The same lentiviral vector encoding GFP alone (Lv-GFP) was used as a control. Cerebellar organotypic cultures were infected with Lv-SCLIP just after plating and fixed after 3,7 , and 14 div to evaluate SCLIP-myc expression in PCs by immunocytochemistry. Myc immunolabeling revealed no detectable SCLIP expression at 3 div and only weakly detectable levels after 7 div in infected slices (data not shown). SCLIP-myc expression became clearly detected in PCs after 14 div (Fig. 8A), indicating that a certain delay after infection was necessary for SCLIPmyc to be expressed at significant levels in transduced PCs. As observed for endogenous SCLIP (Fig. 3), transduced SCLIPmyc was accumulated in the PC perinuclear Golgi region (Fig. 8A, arrowheads) and detected at punctuate structures along dendritic processes at $14 \mathrm{div}$ (arrows), indicating that overexpression did not modify the specific intracellular localization of the protein.

We then analyzed PC differentiation stages at 14 div in cerebellar organotypic cultures infected with Lv-SCLIP or LvGFP just after plating (Fig. 8). A significant increase $\left(\chi^{2}=11.37 ; p<0.01\right)$ in the proportion of stage 3 cells was observed among PCs transduced with the $\operatorname{Lv}$-SCLIP $(68.2 \pm 0.1 \%)$ compared with the Lv-GFP vector $(57.0 \pm 1.9 \%)$ (Fig. $8 B$ ). This increase was accompanied by a parallel decrease of transduced PCs in stage 2 from $11.5 \pm 3.6 \%$ (Lv-GFP) to $3.5 \pm 0.5 \%$ (Lv-SCLIP). Overexpression of SCLIP thus appeared to promote PC dendritic development. To determine whether SCLIP also stimulated dendritic morphogenesis, we quantified the number of primary dendrites emerging from the soma as well as the number of primary branches along dendrites of transduced PCs in stage 3. As revealed in Figure $6, C$ and $D$, both parameters were slightly but significantly increased in Lv-SCLIP transduced PCs compared with control, Lv-GFP transduced cells. The number of primary dendrites averaged $4.26 \pm 0.11$ in PCs overexpressing SCLIP versus $3.93 \pm 0.13$ in control cells, whereas that of primary branches per cell was enhanced from $7.06 \pm 0.34$ to $8.55 \pm 0.42$.

Together, these observations reveal that SCLIP overexpression promotes $\mathrm{PC}$ dendritic development at stage 3 , enhancing both dendritic outgrowth and branching. 


\section{Discussion}

Cerebellar PCs elaborate a complex and highly branched dendritic arbor conferring them an essential integrative function within the cerebellar circuitry. Their dendritic differentiation involves early and late developmental stages characterized by dynamic regressive and growth processes that are tightly regulated (Ramón y Cajal, 1911; Armengol and Sotelo, 1991; Boukhtouche et al., 2006). In this study, we examined the biological properties and functions of the stathmin family protein SCLIP during PC dendritic development. We show that SCLIP specifically accumulates in the growing dendrites of PCs during cerebellar development. By inhibiting its expression in PCs in their tissue environment, we demonstrate that SCLIP is crucial for both the formation and development of PC dendritic arbors at early and late stages of postnatal dendritogenesis.

SCLIP-depleted PCs in P0 cerebellar slices display a round morphology without any dendritic process emerging from the cell body. This dramatic absence of dendritic arborizations at 7 and 14 div is not reflecting poor health or death of PCs after LvshSCLIP lentiviral transduction, because a large number of PCs is still observed after 14 div and some of them even elongate short fingered processes. Moreover, transduced PCs still bear an axon whose morphology appears normal and preserved. However, the absence of dendritic processes is likely the direct consequence of SCLIP depletion in the transduced PCs and not in surrounding other cell types. Indeed, the large majority of transduced cells infected at $\mathrm{P} 0$ are PCs (Fig. 4A) (Boukhtouche et al., 2006). Furthermore, the neighboring untransduced PCs

within the same cellular environment are not affected, and exhibit a normal morphology with protrusions at stage 2 and dendritic processes at stages 3 and 4 . Finally, the first stages of PC dendritic development involving the formation of early dendritic protrusions, their regression, and the subsequent outgrowth and elongation of novel dendritic arborizations have been proposed to occur cell autonomously instead of depending on interactions with neighboring cells (Baptista et al., 1994; Kapfhammer, 2004; Sotelo, 2004). SCLIP thus appears as an important intrinsic PC factor involved in dendritic formation, during both early and later phases of postnatal development.

SCLIP regulates early PC dendritogenesis by controlling both regressive and growth events. Its depletion at an embryonic stage accelerates the retraction of the long primitive process of stage 1 bipolar PCs in addition to prevent the later development of perisomatic protrusions developed by PCs at stage 2 . Only few intracellular factors regulating these early processes have been identified. The transcription factor $\operatorname{ROR} \alpha$ (retinoid-related orphan receptor $\alpha$ ) has recently been shown to control the regressive and remodeling steps of early PC dendritogenesis (Boukhtouche et al., 2006), but the molecular mechanisms underlying its functions remain unknown. The identification of SCLIP as a crucial

B
GFP hless st. 3 chless st. 3
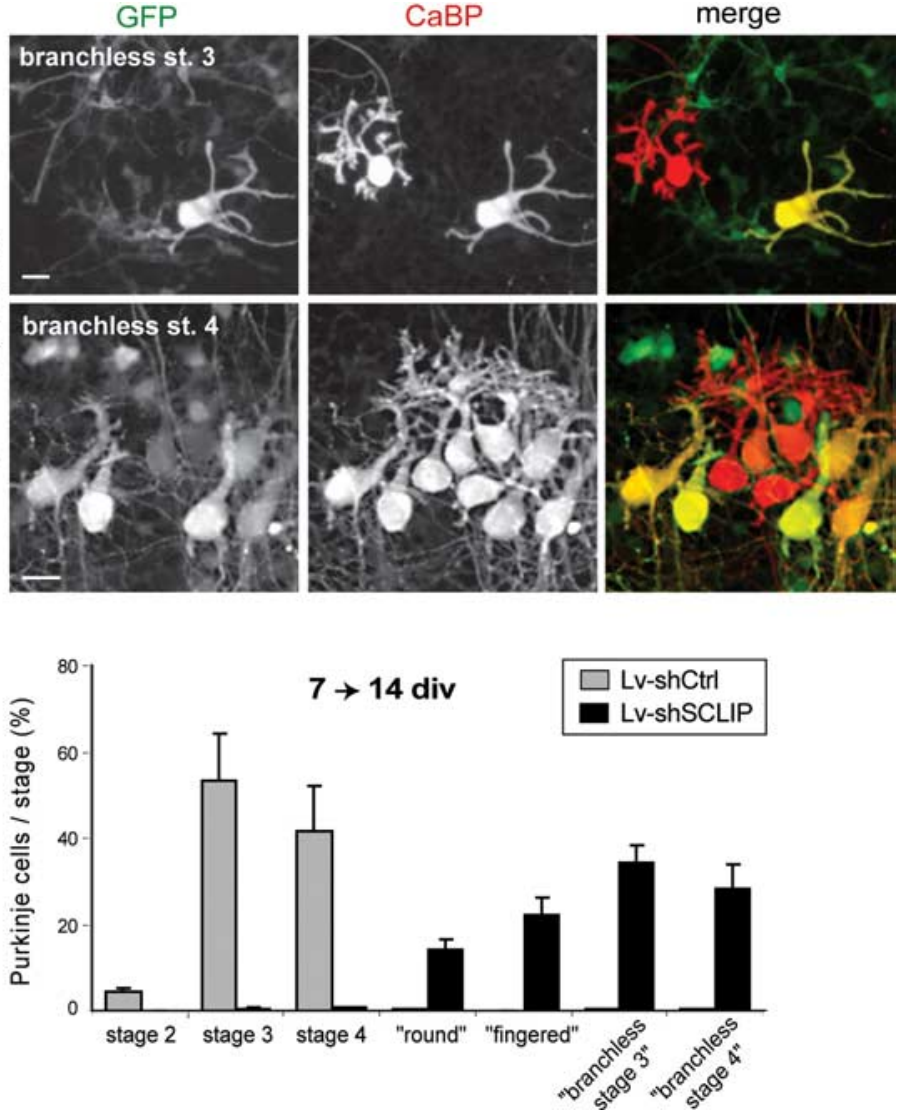

Figure 7. Later SCLIP inhibition blocks $\mathrm{PC}$ dendrite elongation and branching at 14 div. $A$, Examples of $\mathrm{PC}$ morphologies observed at 14 div after infection with Lv-shSCLIP at 7 div. Cerebellar organotypic cultures were infected at 7 div and immunostained for GFP (green) and CaBP (red) 7 later. Whereas untransduced PCs (red) are typically in stages 3 (a) and 4 (b), PCs anchless stage 3 or branchless stage 4, respectively. The remaining PCs exhibit a round or fingered morphology as that observed at 14 div after early RNAi. Values shown are mean \pm SEM.

factor regulating PC process retraction and dendritic outgrowth thus constitutes an important step to better characterize the various intracellular pathways regulating early PC postnatal development. SCLIP activity might indeed be differently solicited, contributing to the control of primitive process integrity and/or its subsequent retraction as well as outgrowth of new dendrites.

Similarly, SCLIP appears also crucial for the later phases of dendritic development. Depleting SCLIP after 7 div in P0 organotypic slices resulted in the formation of "branchless" dendritic arbors at 14 div, indicating that the outgrowth of secondary branches was impaired. Conversely, increasing SCLIP levels promoted dendritic outgrowth and branching of stage 3 PCs. The fact that SCLIP RNAi after 7 div does not prevent the outgrowth of primary dendrites might result from a delay needed for lentiviral-mediated RNAi to knockdown SCLIP in transduced PCs, including the time for degradation of preexisting SCLIP. This delay would allow the formation of primary dendrites, but the subsequent lack of SCLIP would then prevent the outgrowth and elongation of secondary branches. Interestingly, in contrast with its promoting effect on primitive process retraction at stage 1 , the absence of SCLIP does not induce the complete retraction of primary dendrites at stages 3 and 4 . This difference might stem from the fact that, during development, only the primitive process of bipolar PCs retracts in vivo, and that such retraction 
A
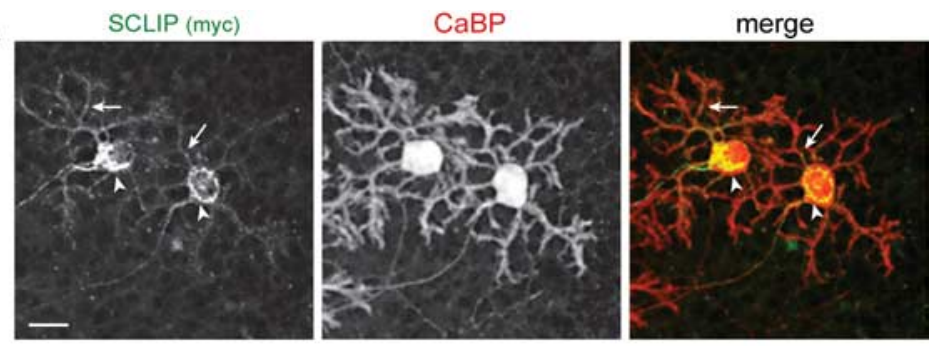

B
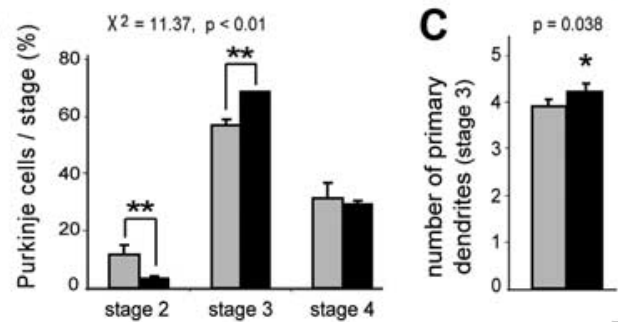

D

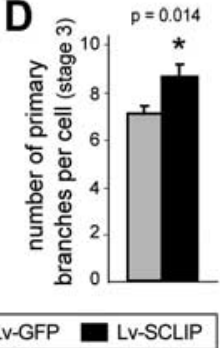

Figure 8. SCLIP overexpression promotes PC dendritic development at stage 3 at 14 div. $\boldsymbol{A}$, Lentiviral-mediated SCLIP-myc transduction allows SCLIP overexpression in PCs. Cerebellar organotypic cultures were infected with the Lv-SCLIP vector just after plating and immunostained for myc (green) and CaBP (red) $14 \mathrm{~d}$ later. A strong labeling corresponding to SCLIP-myc is detected as expected in the perinuclear Golgi region (arrowheads) and dendritic processes (arrows) of transduced PCs. Scale bar, $20 \mu \mathrm{m} . \boldsymbol{B}$, Quantified results of three combined independent experiments illustrating the effect of SCLIP overexpression on PC developmental stages at 14 div. A higher proportion of PCs in stage 3 is observed after infection with Lv-SCLIP compared with LV-GFP. Concomitantly, the proportion of PCS in stage 2 is similarly decreased. Shown are mean values \pm SEM. Differences in the proportions of $P\left(s\right.$ in the various stages are statistically significant ${ }^{* *} p<0.01 ; \chi^{2}$ independence test). C, $D$, Quantified results of three combined independent experiments illustrating the effect of SCLIP overexpression on PC dendrite morphology at stage 3. PCs transduced with Lv-SCLIP vector display an increased number of primary dendrites emerging from the soma $(\boldsymbol{C})$ as well as an increased number of primary dendritic branches $(\boldsymbol{D})$. Values shown are mean $\pm \mathrm{SEM}\left({ }^{*} p<0.05\right.$; Mann-Whitney test).

does not take place for the dendritic tree of mature PCs in normal conditions. SCLIP would then not be required for maintaining the robust dendritic trunk once it has developed, but instead would regulate the retraction and growth of dynamic dendritic processes.

Thus, SCLIP appears essential for the regulation of early and late $\mathrm{PC}$ dendritic differentiation, controlling the retraction of bipolar PC primitive process at stage 1, the outgrowth of perisomatic processes in stellate stage $2 \mathrm{PCs}$, as well as the growth of primary dendrites and secondary branches at stages 3 and 4 . At later stages, interactions with granule cell parallel fibers are known to contribute to the functional maturation of the complex PC dendritic tree. One could speculate that SCLIP might then also play a role, for instance in the transduction of the resulting intercellular signaling.

Interestingly, the crucial regulatory role of SCLIP in PC dendritic development appears quite specific, because it has not been observed for other stathmin family members. Stathmin depletion in cultured PCs did not affect the outgrowth or morphology of dendritic trees (Ohkawa et al., 2007). It only resulted in a $25 \%$ reduction in the total dendritic length, indicating that stathmin might rather play a global modulatory role in late PC dendritic development. Such different functions might stem in part from the different and specific expressions of SCLIP and stathmin in the developing cerebellum. Our study indeed revealed an enrichment of SCLIP in the Purkinje cell layer throughout rat cerebellum development, which has also been observed in the adult rat for SCLIP mRNA (Ozon et al., 1999). In contrast, stathmin seems more widely expressed, because it is mainly detected in the external and internal granular layers from the first postnatal week to the adult age and in the white matter at later phases of development (Himi et al., 1994; Sugiura and Mori, 1995; Ozon et al., 1999). The high expression of SCLIP in PCs compared with stath- min might thus be partially responsible for its specific and essential function in the formation of their dendritic arbors. In addition to their different distributions in the cerebellum, SCLIP and stathmin further display distinct intracellular localizations in PCs that might underlie their functional diversity. As expected from their previously characterized subcellular localization in neurons (Di Paolo et al., 1997; Gavet et al., 2002; Charbaut et al., 2005), stathmin has a homogeneous cytosolic distribution in the soma and dendrites (Ohkawa et al., 2007), whereas SCLIP specifically accumulates at the Golgi complex and vesicular structures in the principal trunk and secondary branches of dendrites during their outgrowth. It is thus tempting to speculate that the specific targeting of SCLIP in growing dendrites might be responsible for its crucial role in PC dendritic outgrowth during cerebellar development. Such a localization appears all the more important because dendritic growth has recently been shown to depend in part on the specific targeting of Golgi outposts in developing dendrites (Ye et al., 2007).

By identifying SCLIP as an essential factor for PC dendritogenesis, our study further demonstrates that SCLIP fulfills various and different functions during neuronal differentiation. We have recently shown that SCLIP participates in the control of the formation of collateral protrusions leading to axonal branching in hippocampal neurons (Poulain and Sobel, 2007). However, these different functions may vary according to the neuronal type and the phase of morphogenesis. Whereas SCLIP depletion accelerated the retraction of primitive processes and prevented the outgrowth of primary dendrites and branches in PCs, it instead promoted the formation of new branches in hippocampal axons. Such opposite functions might stem from different mechanisms involving SCLIP molecular properties. For instance, SCLIP might differently regulate microtubules and their dynamics depending on its phosphorylation state in different neurons or in axonal and dendritic compartments. Phosphorylation has been shown to tightly control the interaction of stathmin family proteins with tubulin (Curmi et al., 1997; Steinmetz et al., 2001; Amayed et al., 2002; Honnappa et al., 2006) and seems necessary to mediate the regulatory functions of stathmin and SCG10 in axonal development (Tararuk et al., 2006; Watabe-Uchida et al., 2006). Alternatively, SCLIP might interact with specific axonal or dendritic partners mediating its functions. SCLIP has indeed been shown to interact with and inhibit the neurite outgrowth-promoting activity of RasGFR1, an exchange factor highly expressed in the cerebellum and strongly detected at synapses (Sturani et al., 1997; Zippel et al., 1997; Baldassa et al., 2007). Characterizing the molecular mechanisms underlying the different functions of SCLIP during the various phases of neuronal morphogenesis and at different cellular regions will thus be of high interest for a better understanding of their regulation throughout development.

In conclusion, SCLIP now emerges as a crucial regulator of neuronal morphogenesis essential for nervous system formation. In particular, its specific regulatory role in the formation and 
development of PC dendritic arborizations is even more remarkable because no intracellular factor regulating the outgrowth of PC dendrites had been identified to date. Defects in SCLIP expression and/or activity might be responsible for pathological conditions in which the development of PC dendritic trees is impaired during cerebellum development.

\section{References}

Altman J (1972) Postnatal development of the cerebellar cortex in the rat. II. Phases in the maturation of Purkinje cells and of the molecular layer. J Comp Neurol 145:399-463.

Amayed P, Pantaloni D, Carlier MF (2002) The effect of stathmin phosphorylation on microtubule assembly depends on tubulin critical concentration. J Biol Chem 277:22718-22724.

Armengol JA, Sotelo C (1991) Early dendritic development of Purkinje cells in the rat cerebellum. A light and electron microscopic study using axonal tracing in "in vitro" slices. Brain Res Dev Brain Res 64:95-114.

Baldassa S, Gnesutta N, Fascio U, Sturani E, Zippel R (2007) SCLIP, a microtubule-destabilizing factor, interacts with RasGRF1 and inhibits its ability to promote Rac activation and neurite outgrowth. J Biol Chem 282:2333-2345.

Baptista CA, Hatten ME, Blazeski R, Mason CA (1994) Cell-cell interactions influence survival and differentiation of purified Purkinje cells in vitro. Neuron 12:243-260.

Belmont LD, Mitchison TJ (1996) Identification of a protein that interacts with tubulin dimers and increases the catastrophe rate of microtubules. Cell 84:623-631.

Boukhtouche F, Janmaat S, Vodjdani G, Gautheron V, Mallet J, Dusart I, Mariani J (2006) Retinoid-related orphan receptor alpha controls the early steps of Purkinje cell dendritic differentiation. J Neurosci 26:1531-1538.

Brochu G, Maler L, Hawkes R (1990) Zebrin II: a polypeptide antigen expressed selectively by Purkinje cells reveals compartments in rat and fish cerebellum. J Comp Neurol 291:538-552.

Charbaut E, Curmi PA, Ozon S, Lachkar S, Redeker V, Sobel A (2001) Stathmin family proteins display specific molecular and tubulin binding properties. J Biol Chem 276:16146-16154.

Charbaut E, Chauvin S, Enslen H, Zamaroczy S, Sobel A (2005) Two separate motifs cooperate to target stathmin-related proteins to the Golgi complex. J Cell Sci 118:2313-2323.

Curmi PA, Andersen SS, Lachkar S, Gavet O, Karsenti E, Knossow M, Sobel A (1997) The stathmin/tubulin interaction in vitro. J Biol Chem 272:25029-25036.

Curmi PA, Gavet O, Charbaut E, Ozon S, Lachkar-Colmerauer S, Manceau V, Siavoshian S, Maucuer A, Sobel A (1999) Stathmin and its phosphoprotein family: general properties, biochemical and functional interaction with tubulin. Cell Struct Funct 24:345-357.

Di Paolo G, Lutjens R, Osen-Sand A, Sobel A, Catsicas S, Grenningloh G (1997) Differential distribution of stathmin and SCG10 in developing neurons in culture. J Neurosci Res 50:1000-1009.

Dusart I, Airaksinen MS, Sotelo C (1997) Purkinje cell survival and axonal regeneration are age dependent: an in vitro study. J Neurosci 17:3710-3726.

Gavet O, El Messari S, Ozon S, Sobel A (2002) Regulation and subcellular localization of the microtubule-destabilizing stathmin family phosphoproteins in cortical neurons. J Neurosci Res 68:535-550.

Himi T, Okazaki T, Wang H, McNeill TH, Mori N (1994) Differential localization of SCG10 and p19/stathmin messenger RNAs in adult rat brain indicates distinct roles for these growth-associated proteins. Neuroscience 60:907-926.

Honnappa S, Jahnke W, Seelig J, Steinmetz MO (2006) Control of intrinsically disordered stathmin by multisite phosphorylation. J Biol Chem 281:16078-16083.

Kapfhammer JP (2004) Cellular and molecular control of dendritic growth and development of cerebellar Purkinje cells. Prog Histochem Cytochem 39:131-182

Naldini L, Blömer U, Gage FH, Trono D, Verma IM (1996) Efficient transfer, integration, and sustained long-term expression of the transgene in adult rat brains injected with a lentiviral vector. Proc Natl Acad Sci U S A 93:11382-11388.

Ohkawa N, Fujitani K, Tokunaga E, Furuya S, Inokuchi K (2007) The mi- crotubule destabilizer stathmin mediates the development of dendritic arbors in neuronal cells. J Cell Sci 120:1447-1456.

Ozon S, Maucuer A, Sobel A (1997) The stathmin family-molecular and biological characterization of novel mammalian proteins expressed in the nervous system. Eur J Biochem 248:794-806.

Ozon S, Byk T, Sobel A (1998) SCLIP: a novel SCG10-like protein of the stathmin family expressed in the nervous system. J Neurochem 70:2386-2396.

Ozon S, El Mestikawy S, Sobel A (1999) Differential, regional, and cellular expression of the stathmin family transcripts in the adult rat brain. J Neurosci Res 56:553-564.

Poulain FE, Sobel A (2007) The "SCG10-Like Protein" SCLIP is a novel regulator of axonal branching in hippocampal neurons, unlike SCG10. Mol Cell Neurosci 34:137-146.

Ramón y Cajal S (1911) Histologie du Système Nerveux de l'Homme et des Vertébrés. Paris: Maloine Edition.

Ramón y Cajal S (1926) Sur les fibres moussues et quelques points douteux de la texture de l'écorce cérébelleuse. Trab Lab Invest Biol Univ Madrid 24:215-251.

Ravelli RB, Gigant B, Curmi PA, Jourdain I, Lachkar S, Sobel A, Knossow M (2004) Insight into tubulin regulation from a complex with colchicine and a stathmin-like domain. Nature 428:198-202.

Sirven A, Ravet E, Charneau P, Zennou V, Coulombel L, Guétard D, Pflumio F, Dubart-Kupperschmitt A (2001) Enhanced transgene expression in cord blood CD34(+)-derived hematopoietic cells, including developing $\mathrm{T}$ cells and NOD/SCID mouse repopulating cells, following transduction with modified trip lentiviral vectors. Mol Ther 3:438-448.

Sobel A (1991) Stathmin: a relay phosphoprotein for multiple signal transduction? Trends Biochem Sci 16:301-305.

Sotelo C (2004) Cellular and genetic regulation of the development of the cerebellar system. Prog Neurobiol 72:295-339.

Steinmetz MO, Kammerer RA, Jahnke W, Goldie KN, Lustig A, van Oostrum J (2000) Op18/stathmin caps a kinked protofilament-like tubulin tetramer. EMBO J 19:572-580.

Steinmetz MO, Jahnke W, Towbin H, García-Echeverría C, Voshol H, Müller D, van Oostrum J (2001) Phosphorylation disrupts the central helix in Op18/stathmin and suppresses binding to tubulin. EMBO Rep 2:505-510.

Sturani E, Abbondio A, Branduardi P, Ferrari C, Zippel R, Martegani E, Vanoni M, Denis-Donini S (1997) The Ras guanine nucleotide exchange factor CDC25Mm is present at the synaptic junction. Exp Cell Res 235:117-123.

Sugiura Y, Mori N (1995) SCG10 expresses growth-associated manner in developing rat brain, but shows a different pattern to p19/stathmin or GAP-43. Brain Res Dev Brain Res 90:73-91.

Tararuk T, Ostman N, Li W, Björkblom B, Padzik A, Zdrojewska J, Hongisto V, Herdegen T, Konopka W, Courtney MJ, Coffey ET (2006) JNK1 phosphorylation of SCG10 determines microtubule dynamics and axodendritic length. J Cell Biol 173:265-277.

Watabe-Uchida M, John KA, Janas JA, Newey SE, Van Aelst L (2006) The Rac activator DOCK7 regulates neuronal polarity through local phosphorylation of stathmin/Op18. Neuron 51:727-739.

Wehrlé R, Caroni P, Sotelo C, Dusart I (2001) Role of GAP-43 in mediating the responsiveness of cerebellar neurons to axotomy. Eur J Neurosci 13:857-870.

Ye B, Zhang Y, Song W, Younger SH, Jan LY, Jan YN (2007) Growing dendrites and axons differ in their reliance on the secretory pathway. Cell 130:717-729.

Zennou V, Serguera C, Sarkis C, Colin P, Perret E, Mallet J, Charneau P (2001) The HIV-1 DNA flap stimulates HIV vector-mediated cell transduction in the brain. Nat Biotechnol 19:446-450.

Zippel R, Gnesutta N, Matus-Leibovitch N, Mancinelli E, Saya D, Vogel Z, Sturani E, Renata Z, Nerina G, Noa ML, Enzo M, Daniella S, Zvi V, Emmapaola S (1997) Ras-GRF, the activator of Ras, is expressed preferentially in mature neurons of the central nervous system. Brain Res Mol Brain Res 48:140-144.

Zufferey R, Nagy D, Mandel RJ, Naldini L, Trono D (1997) Multiply attenuated lentiviral vector achieves efficient gene delivery in vivo. Nat Biotechnol 15:871-875.

Zufferey R, Donello JE, Trono D, Hope TJ (1999) Woodchuck hepatitis virus posttranscriptional regulatory element enhances expression of transgenes delivered by retroviral vectors. J Virol 73:2886-2892. 